Conclusion Susceptibility to nausea is associated with subcortical nuclei morphology. We illustrate a strong association between the ANS and these structural differences. Given these morphological changes are arguably sympathetic-driven, future research should investigate methods to modulate autonomic tone via these nuclei, in order to establish therapies to diminish nausea susceptibility.

\section{PWE-145 THE ROLE OF A GLUTEN FREE DIET IN 'LIFESTYLERS'? THE FIRST DOUBLE BLIND RANDOMISED STUDY}

${ }^{1}$ Anupam Rej ${ }^{*},{ }^{1}$ Matthew Kurien, ${ }^{2}$ Paola Tosi, ${ }^{1}$ Nick Trott, ${ }^{1}$ David Sanders. ${ }^{1}$ Academic Unit of Gastroenterology, Royal Hallamshire Hospital, Sheffield Teaching Hospitals NHS Foundation Trust, Sheffield, UK; ${ }^{2}$ School of Agriculture Policy and Development, University of Reading, Reading,UK

\subsection{6/gutjnl-2018-BSGAbstracts.439}

Introduction A gluten free diet (GFD) is essential in the management of coeliac disease, as well as several studies demonstrating its utility as a dietary therapy in patients with irritable bowel syndrome. The aim of this double-blind placebo-controlled study was to assess the role of a GFD in a healthy population who take a GFD as a lifestyle choice ('lifestylers'). Methods Subjects were recruited via an advert, following exclusion criteria including coeliac disease. Following selection, subjects were commenced on a 2 week GFD following evaluation by a dietitian. Participants were then randomised to receive either organic gluten (Group A, Vital Gluten $14 \mathrm{~g}$ gluten protein/day) or gluten free flour (Group B) in pre-made bags, over a 2 week period. These were sprinkled on their food twice daily. Gastrointestinal Symptom Rating Scale (GSRS) scores were assessed at baseline (following 2 weeks GFD) and after 2 weeks of randomization. Data was analysed using SPSS version 22.

Results 45 subjects were identified with 28 participants recruited into the trial (Group A; $\mathrm{n} \geq 14$, Group B; $\mathrm{n} \geq 14$ ) following exclusion criteria. Median age was 36.5 years (range: 19-63) and $21(75 \%)$ were female. There was no significant difference in baseline demographics between both groups $(\mathrm{p} \geq 0.54)$. Over a 2 week period there was no significant difference in gastrointestinal symptoms or fatigue in either group, as seen in table 1.

\section{Abstract PWE-145 Table 1}

\begin{tabular}{|c|c|c|c|c|c|c|}
\hline GSRS & $\begin{array}{c}\text { Group A } \\
\text { (Baseline) }\end{array}$ & $\begin{array}{l}\text { Group A (End of } \\
\text { Intervention) }\end{array}$ & & $\begin{array}{c}\text { Group B } \\
\text { (Baseline) }\end{array}$ & $\begin{array}{l}\text { Group B (End of } \\
\text { Intervention) }\end{array}$ & \\
\hline & Mean +1 - SO & Mean +1. SD & \begin{tabular}{|c} 
Difference from \\
Baseline (Paired T- \\
test), p-value
\end{tabular} & Mean +1/-SO & Mean $\$ /$ - SO & \begin{tabular}{|c} 
Difference from \\
Baseline (Paired T- \\
test), p-value
\end{tabular} \\
\hline Abdo Pain & $2.50+1 \cdot 1.40$ & $2.14+/ \cdot 1.70$ & 0.504 & \begin{tabular}{|l|}
$2.35+/ 61.33$ \\
\end{tabular} & $2.07+/ \cdot 0.99$ & 0.486 \\
\hline Reflux & $1.71+/ \cdot 1.13$ & $1.64+/ \cdot 1.15$ & 0.72 & $2.50+/ \cdot 2.20$ & $2.57+/ .1 .95$ & 0.895 \\
\hline \begin{tabular}{|l|} 
Indigestion \\
\end{tabular} & $2.14+/ \cdot 1.35$ & $2.07+1 \cdot 1.32$ & 0.876 & $2.14+/ .1 .35$ & $1.79+/ \cdot 0.97$ & 0.336 \\
\hline \begin{tabular}{|l|} 
Diarthoea \\
\end{tabular} & $2.71+/ \cdot 1.93$ & $1.64+\% \cdot 0.92$ & 0.03 & $1.85+/ \cdot 1.46$ & $1.86+/ \cdot 1.35$ & 1 \\
\hline Constipation & $2.50+/ .1 .82$ & $2.46+/ \cdot 1.81$ & 0.697 & $1.92+/ .1 .54$ & $2.50+1 \cdot 1.65$ & 0.179 \\
\hline & & & & & & \\
\hline Fatigue Score & $6.61+/ \cdot 2.36$ & $6.00+/ \cdot 2.98$ & 0.585 & $6.57+/-2.44$ & $5.36+/-2.27$ & 0.232 \\
\hline
\end{tabular}

Conclusion This study demonstrates that gluten is unlikely to be the culprit agent for gastrointestinal symptoms or fatigue in healthy individuals. A GFD has no evidence base in individuals who do not have coeliac disease or IBS. The public should be discouraged from considering a GFD of their own volition.
PWE-146 PAIN ENDOPHENOTYPES DISPLAY COMPLEX SUBCORTICAL BRAIN MORPHOLOGICAL DIFFERENCES INFLUENCED BY AUTONOMIC NEUROPHYSIOLOGY OR PERSONALITY TRAITS

${ }^{1}$ James Ruffle*, ${ }^{2}$ Steve Coen, ${ }^{3}$ Vincent Giampietro, ${ }^{3}$ Steven Williams, ${ }^{1}$ Qasim Aziz, ${ }^{1,4}$ Adam Farmer. ${ }^{1}$ Centre for Neuroscience and Trauma, Blizard Institute, Wingate Institute of Neurogastroenterology, Barts and the London School of Medicine and Dentistry, Queen Mary University of London, London, UK; ${ }^{2}$ Research Department of Clinical, Educational and Health Psychology, University College London, London, UK; ${ }^{3}$ King's College London, Institute of Psychiatry, Psychology and Neuroscience, Department of Neuroimaging, London, London, UK; ${ }^{4}$ Department of Gastroenterology, University Hospitals Midlands NHS Trust, Stoke on Trent, UK

\subsection{6/gutjnl-2018-BSGAbstracts.440}

Introduction Visceral pain is influenced by an array of individual factors. We have previously coalesced many of these, reporting that two major endophenotypic 'pain clusters' exist: Pain Cluster 1 (PC1), in comparison to Pain Cluster 2 (PC2), had higher neuroticism and anxiety scores, higher baseline sympathetic tone and serum cortisol, but during acute pain had a lower stimulus tolerance and increased parasympathetic tone. Meanwhile, PC2 had the converse profile at baseline and during pain. Endophenotypes were reproducible if reassessed annually, and the two most influential factors in allocating PCs were personality trait neuroticism and autonomic tone. We therefore hypothesised that PCs could linked to altered subcortical morphology.

Methods Endophenotype were determined in 27 healthy subjects (14 male; mean age 30 years, PC1 $(n \geq 11)$ and PC2 $(\mathrm{n} \geq 16)$, and all underwent structural neuroimaging. Subcortical morphological changes were studied contingent on PC. By alternate regression of PC factors, autonomics and personality traits, we analysed if PC associated brain morphology was affected by autonomic or personality-driven effects.

Results Neuroticism-driven effect PC1 subjects exhibited morphological deformation differences localised to the right amygdala and pallidum, when contrasted to PC2 subjects with effect of autonomic tone regressed. Autonomic-driven effect: Sympathetic tone (cardiac sympathetic index), with regression of personality trait neuroticism, was positively linearly associated with structural deformation changes at the left pallidum, right nucleus accumbens and right putamen (figure 1).

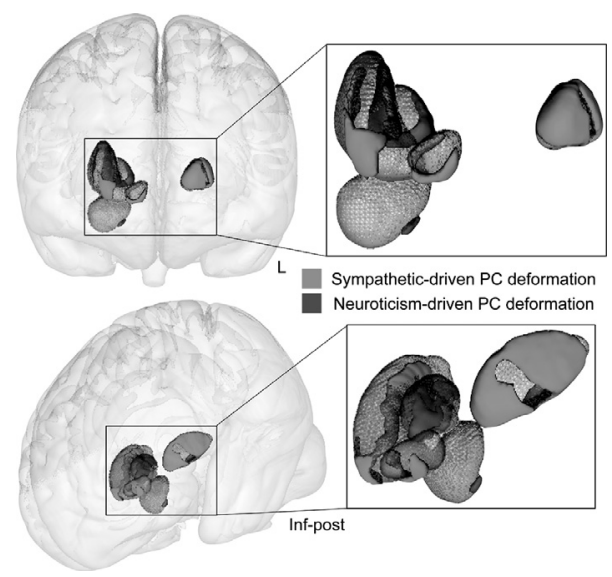

Abstract PWE-146 Figure 1 Subcortical morphology is associated with endophenotypic pain clusters. Sympathetic-driven effects (blue) [L pallidum, $R$ accumbens and $R$ putamen] in addition to neuroticismdriven effects (red) [R amygdala and $R$ pallidum]. 
Conclusions Personality and autonomic neurophysiology influences subcortical morphological changes identified in PCs. Future research should investigate associations of endophenotypic characteristics to brain structure and function in health and chronic visceral pain to establish biomarkers for personalised medicine-based approaches.

\section{PWE-147 SYSTEMATIC STUDY OF GASTROINTESTINAL SYMPTOMS AND QUALITY OF LIFE IN CYSTIC FIBROSIS: THE 'CF GUT'}

\author{
${ }^{1}$ Bu'Hussain Hayee*, ${ }^{1}$ Kerry-Lee Watson, ${ }^{1}$ Emma Howard, ${ }^{1}$ Penelope Hutchings, \\ ${ }^{2}$ Sanchika Campbell, ${ }^{2}$ Anna Simpson, 'Caroline Elston. 'King's College Hospital, London, \\ UK; ${ }^{2}$ King's College London, UK
}

\subsection{6/gutjnl-2018-BSGAbstracts.441}

Introduction The 'CF gut' is a novel term encompassing the range of gastrointestinal (GI) symptoms recognised in patients with cystic fibrosis (CF). There are no accepted or validated assessment tools and neither the range nor the frequency of symptoms has been described. Evidence from our CF-GI clinic demonstrated that symptoms were not accounted for by pancreatic insufficiency (PI) and appeared to correspond to those seen in IBS. These symptoms will impact quality of life (QOL) outside of pulmonary morbidity, therefore it is not clear whether current CF-related QOL tools are sufficient.

Methods Consecutive patients attending specialist CF clinics were asked to complete questionnaires: Patient Health (PHQ9); Generalised Anxiety (GAD-7); GI symptom rating scale (GSRS); IBS symptom severity score (IBS-SSS); CF-related quality of life (CFQR). Demographics, BMI, CF genotype, PI status and enzyme replacement therapy (PERT) were recorded. Patients with pre-existing coeliac disease or inflammatory bowel disease were excluded. Questionnaires were altered to remove the term 'IBS' and patients were asked about 'GI symptoms' instead.

Results Results from the total cohort of 176 patients will be forthcoming, but we present interim data from 107 (mean age $27.8 \pm 9.6 \mathrm{y} ; 60 \mathrm{~F}$; 94 PI $(88 \%)$ of whom 2 were not taking PERT; mean BMI $22.1 \mathrm{~kg} / \mathrm{m}^{2}, \quad \mathrm{FEV}_{1} 59 \%$ predicted). 53 (49.5\%) were $\Delta$ F508 homozygous.

69/107 (65\%) met Rome IV criteria for IBS, with 47 (44\%) reporting significant symptoms (IBS-SSS >80). Using the GSRS we created a 'heatmap' to describe the range and severity of symptoms as IBS-SSS increased (figure: 'traffic light' colour chart for mild-moderate-severe, columns 1-11 are each descriptor in the GSRS; rows are individual patients with IBS-SSS increasing down left-most column).

With patients grouped as IBS-SSS $<80$ or $>80$, significant differences were observed in anxiety and depression as well as across all domains of the CFQR. There was no correlation between IBS-SSS and any CFQR domain (particularly that relating to GI symptoms), suggesting that the latter is insufficient to describe the CF gut.

Conclusion This is the first ever systematic study using validated symptom scores to describe the range of GI symptoms in CF. These do not correlate with PERT or genotype and appear to be captured well by the IBS-SSS, but not the CFQR. Further work will be aimed at establishing accurate screening and assessment tools for this phenotype. Therapeutic trials in CF may use these already validated tools to demonstrate a positive impact on 'non-respiratory' symptoms and QOL.

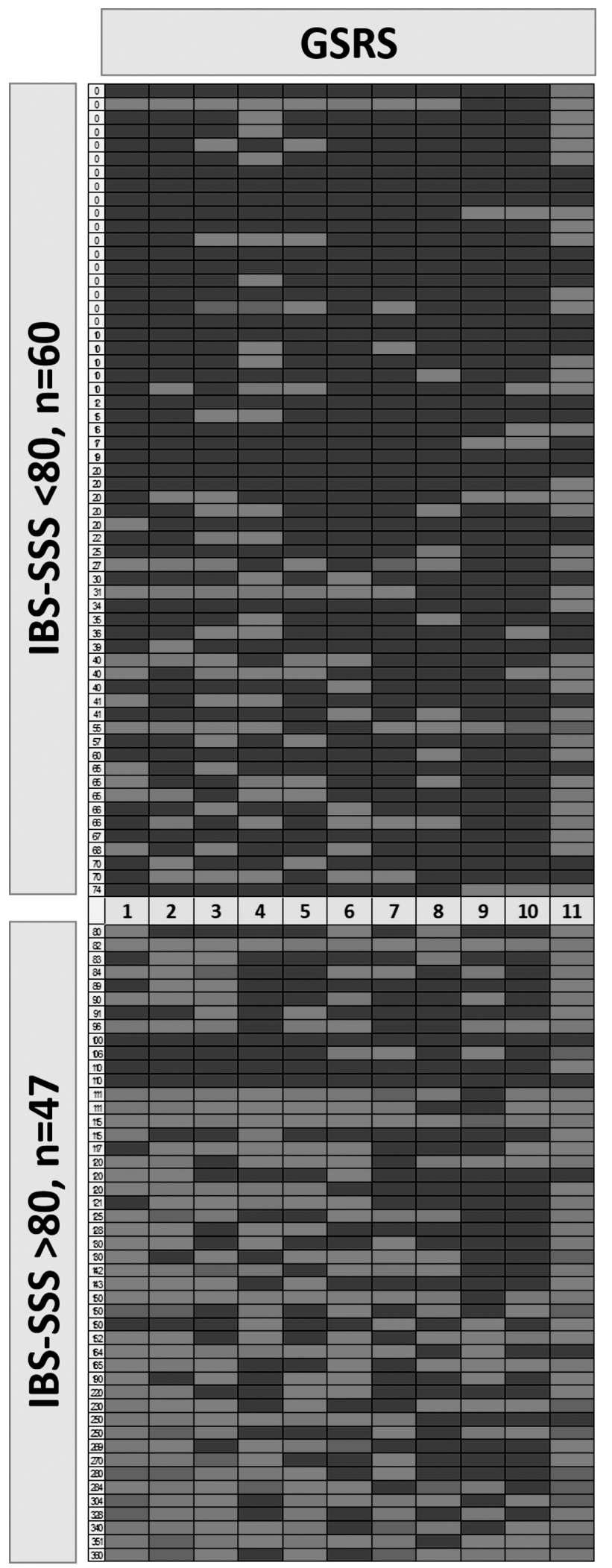

Abstract PWE-147 Figure 1 